\title{
La formación de Enfermería del Trabajo
}

\section{Training in Occupational Health Nursing}

\section{Javier González Caballero}

${ }^{1}$ Instituto Nacional de la Seguridad Social. Servicio de Prevención y Salud Laboral, Bilbao, España.

Fechas · Dates

Recibido: 2020.09 .09

Aceptado: 2020.09.19

Publicado: 2020.11.04

\section{Correspondencia $\cdot$ Corresponding Author}

Javier González Caballero

Servicio de Prevención y Salud Laboral. Instituto Nacional de la Seguridad Social. Gran Vía de Don Diego López de Haro, 62. 48011. Bilbao

Correo electrónico: javier.gonzalez1@seg-social.es 
La enfermería del trabajo cuenta con una extensa trayectoria en el ámbito de la atención especializada por su formación específica y presencia en los centros de trabajo. El proceso de adquisición de competencias profesionales admite distintas particularidades en el ámbito de la Unión Europea, no obstante la especialidad está inmersa en un entorno laboral complejo, diverso y variable donde asoman cambios significativos y nuevos retos para la seguridad y salud en el trabajo(1).

La Comisión Nacional de la Especialidad de Enfermería del Trabajo definió en 2009 unas competencias propias y un programa formativo específico. Esta circunstancia constituyó en su momento un punto de inflexión en esta disciplina enfermera(2). La adquisición de competencias permite acceder a un nivel de conocimientos, habilidades y actitudes a través de la capacitación en las siguientes áreas: preventiva, asistencial, legal y pericial, gestión, docencia e investigación. En consecuencia, la adscripción a una unidad docente acreditada implica completar un periodo formativo de dos años que incluye contenidos teóricos y prácticos.

En el ámbito comunitario, la Federation of Occupational Health Nurses within the European Union (FOHNEU) describe las competencias de la especialidad en distintos países y analiza los programas docentes impartidos, observando un acceso generalizado a la especialidad a través de un master universitario ${ }^{(3)}$. Aunque en la estructura del programa difiere en función del país, los contenidos relativos a la salud pública y comunitaria tienen un gran peso en todos los $\operatorname{casos}^{(4)}$ (Tabla 1). Aun así, la propia federación profesional señala dos consideraciones: en seis países disponen de contenidos específicos durante el periodo de pregrado y en doce han establecido programas de especialización concretos $^{(5)}$.

De forma especifica, la propia Federation of Occupational Health Nurses within the European Union, la American Association of Occupational Health Nurses, la Canadian Nurses Association, la Association of Occupational Health Nurse Practitioners, el Scientific Committee on Occupational Health Nursing, la Oficina Regional para Europa de la Organización Mundial de la Salud, el Occupational Health Nursing Research Center y la Faculty of Occupational Health Nursing también han definido los distintos roles de la enfermería del trabajo en sus áreas de influencia(6).

En el primer estudio en la Unión Europea donde se analiza la opinión y la percepción de las competencias profesionales de esta disciplina enfermera, los especialistas alcanzan un mayor grado de desarrollo en aquellos cometidos relacionados con el área asistencial. En cambio, consideran más importantes los contenidos relativos a la prevención y promoción de la salud((6), percepciones coincidentes con distintos autores ${ }^{(7)}$. En este relato de prioridades de la especialidad asoman otras circunstancias influyentes: una realidad socioeconómica globalizada, las complejas relaciones laborales en un escenario cambiante, las características del mercado de trabajo actual, las expectativas y actitudes tanto empresariales como de los profesionales, la dimensión de la relación que se establece con la población laboral, la aparición de nuevas enfermedades o patologías emergentes ligadas al trabajo y la herencia de modelos organizativos y asistenciales previos. Todas ellas determinan el desarrollo y la ejecución de las competencias del colectivo más numeroso que presta cuidados de salud en los centros de trabajo(8). 
Tabla 1. Análisis comparativo de las competencias de enfermería del trabajo en diferentes países europeos.

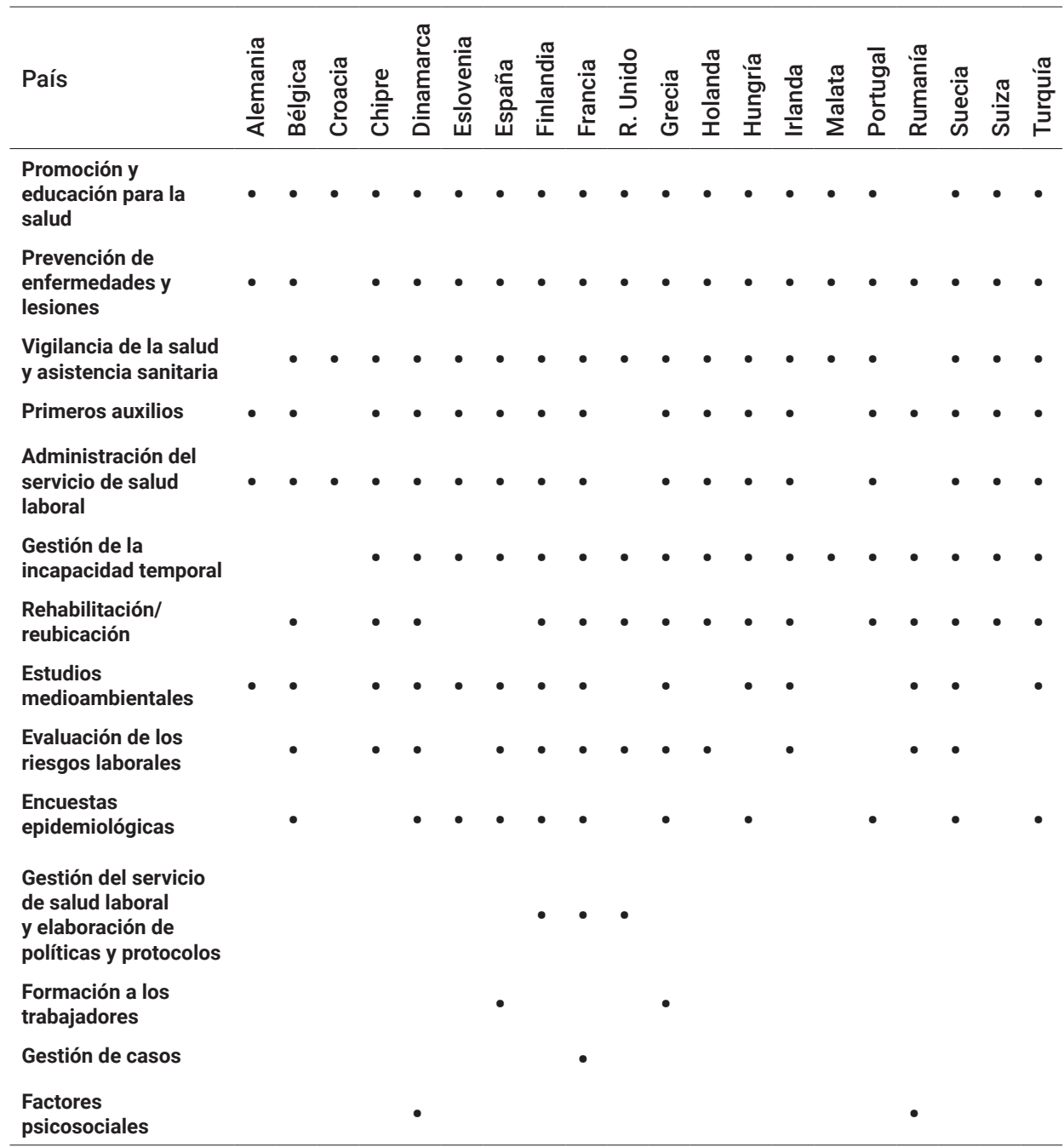

Fuente: Staun J. Occupational Health Nurses status in European Union states 2005-2012. Final results. Federation of Occupational Health Nurses within the European Union (FOHNEU), 2014.

En este recorrido a través de especialidad de enfermería del trabajo se observan fortalezas y debilidades formativas, no obstante el $67 \%$ de los países desarrollados disponen de una titulación oficial y un programa específico de la especiali$\mathrm{dad}^{(8)}$. La American Association of Occupational Health Nurses revisa mediante el método Delphi sus competencias cada cuatro años con un propósito nítido, analizar la práctica profesional de los actores implicados ${ }^{(8)}$. Por tanto, desde una propuesta con enfoque laboral pero cercana y complementaria al ámbito comunitario, se trata de provocar una reflexión sobre la oportunidad de armonizar los contenidos formativos de forma periódica con el fin de consolidar una disciplina que proporcione unos cuidados a la población laboral basados en la mejor evidencia científica disponible. 


\section{Referencias}

1. González J. Análisis de las competencias de Enfermería del Trabajo. Rev Soc Esp Salud Admon Pública. 2016;IIl:11-7.

2. Boletín Oficial del Estado. Orden SAS/1348/2009 de 6 de mayo por la que se aprueba y publica el programa formativo de la especialidad de Enfermería del Trabajo. BOE núm. 129 de 28/5/2009. [Acceso 16 Sep 2020]. Disponible en: http:// www.boe.es/boe/dias/2009/05/28/pdfs/BOE-A-2009-8880.pdf

3. Federation of Occupational Health Nurses in the European Union. Occupational Health Nursing. Education, practice and profile in the EU countries. Education Group. Final report, 2012 [acceso 18 Jun 2020]. Disponible en: https://fohneu.org/ images/pdf/final-report-on-OHN-education-practice-and-profile-2012.pdf

4. Federation of Occupational Health Nurses in the European Union. Occupational Health Nursing. Education and practice in the EU countries. Education Group, FOHNEU Final report 2014 [acceso 16 Sep 2020]. Disponible en: https://fohneu.org/ images/pdf/final-report-on-OHN-education-practice-and-profile-2012.pdf

5. Staun J. Occupational Health Nurses status in European Union states 20052012. Final results. Federation of Occupational Health Nurses within the European Union, 2014.

6. González J. Evolución y desarrollo de las competencias profesionales de Enfermería del Trabajo. Bizkaia: Universidad del País Vasco/Euskal Herriko Universitatea; 2017. Disponible en: https://addi.ehu.es/bitstream/handle/10810/30604/ TESIS_GONZALEZ_CABALLERO_JAVIER.pdf?sequence=1\&isAllowed=y

7. Rogers B, Kono K, Palucci MH, Peurala M, Radford J, Staun J. International survey of Occupational Health Nurses'roles in multidisciplinary teamwork in occupational health services. Workplace Health Saf. 2014;62(7):274-81.

8. González J. Estudio sobre las competencias profesionales de Enfermería del Trabajo en España. Arch Prev Riesgos Labor. 2020;23(1):34-51. 